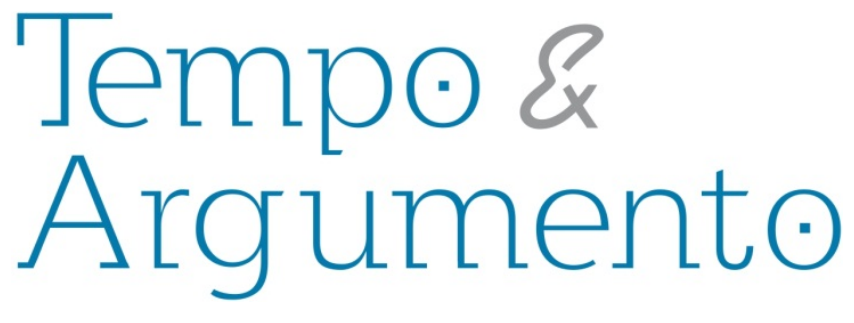

\title{
Les archives de la seconde guerre mondiale en France, de I'histoire à l'histoire de soi
}

\begin{abstract}
Résumé
L'ouverture au public des archives de la seconde guerre mondiale dans les années 1990 a constitué un épisode marquant et parfois conflictuel de l'histoire récente des archives en France. La période a en effet été marquée par une série de débats vifs voire de polémiques sur les conditions juridiques de l'accès à ces documents. Nous revenons ici sur ces années difficiles en nous efforçant de décrypter ce qu'elles ont pu signifier en termes de relation entre lecteur et document d'archives. Derrière un évident besoin d'histoire et une problématique de mémoire sociale et collective qui ne sauraient être niés, se cache aussi une assez profonde transformation des logiques d'usage du document d'archives.
\end{abstract}

Mots-clés : Histoire. Archives. Mémoire. Seconde guerre mondiale. Juifs.

\author{
Patrice Marcilloux \\ Maître de conférences en \\ archivistique. \\ Université d'Angers. \\ patrice.marcilloux@laposte.net
}

\footnotetext{
Pour citer cet article :

MARCILLOUX, Patrice. Les archives de la seconde guerre mondiale en France, de l'histoire à

I'histoire de soi. Revista Tempo e Argumento, Florianópolis, v. 5, n.9, jan./jun. 2013. p. 288 - 311.
}

DOI: $10.5965 / 2175180305092013288$

http://dx.doi.org/10.5965/2175180305092013288 


\section{The second world war \\ archives in France, from history to one's own history}

\section{Os arquivos da segunda guerra mundial na França, da história à história de si}

\begin{abstract}
The public opening of the archives of the Second World War in the 1990s was a remarkable and sometimes conflicted episode in the recent history of archives in France. The period was marked, in effect, by a series of heated and sometimes controversial debates on the juridical conditions for the access to these documents. Here we return to these difficult years, striving to decipher what they could have meant in terms of the relationship between reader and archive documents. Behind an obvious need for history and a problematic social and collective memory that could not be denied, hides a very profound transformation of the logic of use of archive documents.
\end{abstract}

Keywords: History. Archive. Memory. Second world war. Jews.

\section{Resumo}

A abertura ao público dos arquivos da segunda guerra mundial nos anos 1990 constituiu um episódio marcante e por vezes conflituoso da história recente dos arquivos na França. O período foi marcado, com efeito, por uma série de calorosos debates, por vezes polêmicos, sobre as condições jurídicas do acesso a esses documentos. Voltamos aqui a esses anos difíceis, esforçando-nos por decifrar o que eles puderam significar em termos de relação entre leitor e documento de arquivos. Atrás de uma evidente necessidade de história e de uma problemática de memória social e coletiva que não poderiam ser negadas, esconde-se uma assaz profunda transformação das lógicas de uso do documento de arquivos.

Palavras-chave: História. Arquivo. Memória. Segunda guerra mundial. Judeus. 
Les conditions de l'ouverture des archives de la seconde guerre mondiale aux historiens professionnels ou amateurs et au grand public dans les années 1990 constituent une des pages sans doute les plus marquantes de l'histoire récente des archives en France. L'épisode a débouché sur une révision de la législation archivistique en vigueur avec la mise en chantier dès 1996 d'un projet de loi modifiant la loi du 3 janvier 1979, qui ne devait pourtant aboutir qu'en $2008^{1}$. Il a aussi révélé une intensification de la demande sociale adressée à l'administration des archives, dans un climat de tensions voire de polémiques que les archivistes ont parfois vécu comme d'injustes mises en cause. Sans oser encore ici une mise en histoire complète de cette période, laquelle reste à faire ${ }^{2}$, nous voudrions ici attirer l'attention sur un aspect plus méconnu et pourtant fondamental à nos yeux de ce moment singulier dans lequel nombre d'observateurs n'ont

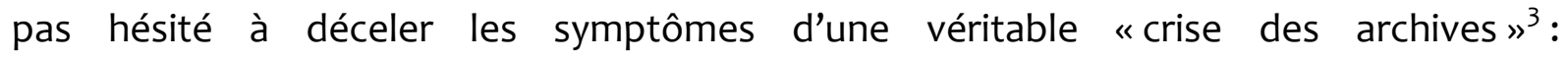
l'individualisation progressive du rapport aux archives sur fond de relativisation de leur usage historique, un passage de l'histoire à l'histoire de soi en quelque sorte. La célèbre affaire dite du fichier juif et la question de l'indemnisation des spoliations des Juifs de France nous serviront de prisme révélateur de cette utilisation différente des archives mélangeant grande et petite histoire, compréhension historienne du passé et stratégies familiales. On ne saurait néanmoins entreprendre cette tâche sans avoir dans un premier temps brièvement rappelé le cadre réglementaire en vigueur dans la décennie $1990 \mathrm{ni}$ sommairement décrit les documents qui sont en cause.

\section{Fonds et communicabilité légale}

Au début des années 1990, cinquante ans après le début de la seconde guerre mondiale, la communication des archives aux différents publics qui désirent y accéder est

1. BÉLAVAL (Philippe), «Archives et République », Le Débat, 2001, n 115, p. 100-117 et «Les enjeux de la réforme de la loi de 1979 ", dans CORNU (Marie), FROMAGEAU (Jérôme), sous la dir., Archives et recherche, aspects juridiques et pratiques administratives, Paris, L'Harmattan, 2003, p. 9-19.

2. DUCLERT (Vincent), «Les enjeux de la politique des archives en France ", dans POIRRIER (Philippe), LAUVERNIER (Julie), sous la dir., Historiographie et archivistique. Écriture et méthodes de l'histoire à l'aune de la mise en archives, Territoires contemporains, nouvelle série, $n^{\circ} 2$.

3. DUCLERT (Vincent), "Les historiens et la crise des archives ", Revue d'histoire moderne et contemporaine, 2001, nं 48, p. 16-43. 
légalement fixée par la loi du 3 janvier 1979, un décret du 3 décembre 1979 venant préciser certaines dispositions de la loi sur le plan réglementaire. L'analyse rapide de ce régime juridique, qui ne concerne que les archives auxquelles la loi reconnaît le caractère d'archives publiques, amène à constater à la fois que beaucoup de documents relatifs à la seconde guerre mondiale ne sont pas encore communicables, que, grâce au système des dérogations, la recherche historique ne connaît pas de véritable obstacle à son développement mais, en même temps, que si l'équilibre défini par le droit positif permet de faire face aux besoins d'histoire, il ne parvient déjà plus à répondre à l'ensemble des demandes adressées au système archivistique par tous ses usagers.

\section{La loi et le décret}

Votée dans le contexte de transparence administrative instauré par la loi du 17 juillet 1978 sur la liberté d'accès aux documents administratifs et la loi du 6 janvier 1978 relative à l'informatique, aux fichiers et aux libertés, la loi de 1979 sur les archives était réputée jusqu'au début des années 1990 avoir instauré un régime "très libéral » ${ }^{4}$ de communication des archives publiques. Rassemblant, uniformisant et complétant des dispositions jusqu'alors éparses et d'origine souvent simplement réglementaire, elle prévoit que tous les documents librement communicables avant leur entrée aux archives le demeurent et que les autres subissent un délai commun de trente ans. Les documents plus confidentiels font exception et sont régis par des délais spéciaux, au nombre de cinq et variant entre soixante et cent cinquante ans.

Les archives de la seconde guerre mondiale sont principalement concernées par deux de ces délais. Un délai de soixante ans à compter de la date de l'acte touche les documents qui contiennent des informations mettant en cause la vie privée ou intéressant la sûreté de l'État ou la Défense nationale et dont la liste est fixée par le décret du 3 décembre 1979. Bien que l'arrêt Jobez du Conseil d’État soit venu en 1994 limiter la portée du décret en ce qui concerne la notion de sûreté de l’État, la pratique

\footnotetext{
4. PONTIER (Jean-Marie), RICCI (Jean-Claude), BOURDON (Jacques), Droit de la culture, Paris, Dalloz, 1990,
} p. 338. 
archivistique courante continue à considérer que la présence d'une catégorie de documents dans la liste donnée par le décret du 3 décembre 1979 suffit ipso acto à la soumettre au délai de 60 ans $^{5}$. Un délai de cent ans à compter de la date de l'acte ou de la clôture du dossier porte sur les documents relatifs aux affaires portées devant les juridictions, y compris les décisions de grâce.

Surtout, reprenant une tradition instaurée dès le XIX ${ }^{\mathrm{e}}$ siècle, la loi de 1979 instaure un régime de dérogations en vertu duquel les documents soumis au délai trentenaire et aux délais spéciaux peuvent bénéficier d'une consultation anticipée, avant l'expiration de ces délais, sur décision du directeur des Archives de France qui statue après accord de l'administration qui a versé les documents. Bien que la loi ait prévu des dérogations individuelles et générales, la pratique archivistique favorise nettement les dérogations individuelles qui, si elles ont l'avantage de permettre assez facilement les recherches à caractère historique, présentent l'inconvénient de nourrir tous les soupçons à l'égard des archivistes suspectés d'arbitraire dans leur perception de la nature des demandes et de leurs motivations ${ }^{6}$. Même la faveur traditionnellement accordée aux demandes de dérogations orientées vers la production d'une recherche historique, toute parée des meilleures intentions qu'elle soit, peut faire problème et être interprétée au sceau de l'élitisme et de la fermeture. La circulaire dite Jospin du 2 octobre 1997 relative à l'accès aux archives publiques de la période 1940-1945 réaffirme ce point de vue, dans un souci pourtant d'ouverture: «Les dérogations devront être largement accordées pour effectuer des recherches historiques, en particulier aux membres de la communauté scientifique ou universitaire (chercheurs et enseignants), qu'ils soient français ou étrangers. Il en ira de même pour les étudiants de l'enseignement supérieur présentant des demandes dans le cadre de la préparation d'un mémoire ou d'une thèse. " ${ }^{7}$

\footnotetext{
5 . BASTIEN (Hervé), Droit des archives, Paris, La Documentation française, 1996, p. 96-97.

6. COMBE (Sonia), Archives interdites : les peurs françaises face à l'histoire contemporaine, Paris, Albin Michel, 1994, $328 \mathrm{p}$.

7. Journal officiel de la République française, 3 octobre 1997, p. 14340.
} 


\section{Les fonds d'archives}

En application de ces dispositions légales et réglementaires se trouvent donc exclus d'une libre communication immédiate en ce début des années 1990 de nombreux documents d'un intérêt certain à la fois pour l'écriture de l'histoire de la seconde guerre mondiale et pour les recherches à caractère généalogique ou familial. Les arrêtés de dérogation générale qui devaient finalement être pris en 1999 et 2002 en témoignent en creux éloquemment quoique partiellement ${ }^{8}$. Pour s'en tenir aux seules archives départementales (arrêté du 29 avril 2002 publié au Journal officiel du 3 mai 2002), ce sont notamment les rapports périodiques des préfets et des sous-préfets, les rapports de police et des renseignements généraux, certains dossiers des cabinets de préfets, notamment ceux relatifs à la surveillance des organisations politiques et syndicales ou à l'organisation des camps d'internement et des convois de déportation, les archives des camps d'internement, les documents à caractère nominatif émanant du Service du travail obligatoire, tous fonds et documents tombant sous le coup du délai de soixante ans. Aux Archives nationales, ce sont en particulier les archives de fort nombreux services dépendant du ministère de l'Intérieur ou versés par le ministère des Anciens Combattants (arrêté du 11 octobre 1999 publié au Journal officiel du 17 novembre 1999 et arrêté du 15 avril 1999 publié au Journal officiel du 13 mai 1999) : cabinet du ministre, Sûreté générale, renseignements généraux, surveillance des partis et mouvements, inspection des camps, fichiers de police, fichiers des camps. Il faut y ajouter toutes les archives issues des juridictions, et notamment les archives des cours de justice créées en 1944 aux fins d'épuration des collaborateurs, qui, elles, devaient rester à l'écart des arrêtés de dérogation générale de la fin des années 1990 et du début des années 2000, pour cause de délai de communicabilité fixé à cent ans.

\footnotetext{
8. Pour une vision d'ensemble : BLANC (Brigitte), ROUSSO (Henri), TOURTIER-BONAZZI (Chantal de), sous la dir., La seconde guerre mondiale. Guide des sources conservées en France. 1939-1945, Paris, La Documentation française, 1994, $1218 \mathrm{p}$.
} 


\section{La demande}

Les chiffres publiés par la direction des Archives de France dans son rapport annuel d'activité montrent d'abord une forte croissance du nombre des demandes de dérogations, globalement considérées : 482 demandes en 1985, 988 en 1990, 3184 en 2001, soit une multiplication par plus de 6 en 15 ans. Même si cette accroissement est à mettre en relation avec l'augmentation très sensible de la fréquentation des archives nationales et départementales, laquelle est multipliée par trois dans les mêmes années, il est difficile de ne pas voir dans ces éléments statistiques le signe sinon d'une remise en cause du régime légal de communicabilité, du moins d'une pression exercée sur lui et comme un indice de son inadaptation.

La répartition de ces demandes par période montre l'écrasante prédominance de la seconde guerre mondiale : 1019 demandes concernent la seconde guerre mondiale en 2001, soit 32\% de toutes les demandes de dérogations introduites cette année-là. À la suite de l'arrêté de 2002 portant ouverture par dérogation générale des archives des services déconcentrés du ministère de l'Intérieur, la part des dérogations liées à la seconde guerre mondiale diminue mais se stabilise à un niveau significatif : $20 \%$ en $2002,14 \%$ en $2003,18 \%$ en $2005,14 \%$ en 2006, $15 \%$ en 2007 . Entre 2005 et 2007 , les rédacteurs du rapport annuel de la direction des Archives de France notent avec constance que « cette période suscite toujours beaucoup de demandes de dérogation ${ }^{9}$. La ventilation des demandes selon leur nature est également riche d'enseignement, même si les chiffres sont ici plus sujets à caution puisque tributaires à la fois de la perception de la nature de sa demande par le pétitionnaire au moment où il remplit le formulaire officiel d'inscription dans un service d'archives et des éventuels correctifs apportés par la direction des Archives de France. On note néanmoins que, en 2002 encore, soit après la publication des arrêtés de dérogation générale, $27 \%$ des demandes portent sur des «recherches historiques personnelles » ou présentent un caractère "généalogique », alors que 71\% concernent des «recherches scientifiques ", selon la terminologie employée par l'administration centrale des archives.

9. Des archives en France, l'activité de la direction des Archives de France et des services publics d'archives, 2005, p. 64 ; Des archives en France, l'activité de la direction des Archives de France et des services publics d'archives, 2006, p. 98 ; Des archives en France, l'activité de la direction des Archives de France et des services publics d'archives, 2007, p. 103. 
"émanent de personnes désireuses de consulter des archives de juridiction soit pour mieux connaître le parcours d'un de leurs ascendants condamné pendant l'occupation ou lors de l'épuration, soit, dans une moindre proportion, pour mener, individuellement ou dans un cadre associatif, des recherches sur la Résistance et la Collaboration ${ }^{10}$.

\section{Fichier juif et CIVS : un apparent besoin d'histoire}

Deux ouvrages ${ }^{11}$, une trentaine d'articles dans un journal comme Le Monde, des milliers de pages Web, l'affaire dite du fichier juif a exprimé entre 1991 et 1997 de manière paroxysmique la prise de conscience par le grand public de la responsabilité de l'État français dans la persécution des Juifs de France pendant la seconde guerre mondiale. Elle met aussi au jour la complexité du rapport entre archives, histoire, mémoire, société et usages individuels. Créée par décret du Premier ministre le 10 septembre 1999, la Commission pour l'indemnisation des victimes de spoliations intervenues du fait des législations antisémites en vigueur pendant l'occupation (CIVS) en prend acte dans sa pratique. Ici, le besoin d'histoire n'est pas premier ; il résulte d'une somme de demandes individuelles à caractère initialement juridique et financier.

\section{L'affaire du fichier juif : repères chronologiques}

Le 13 novembre 1991, un article du journal Le Monde révèle que Serge Klarsfeld, avocat de certaines parties civiles dans la procédure de crime contre l'humanité ouverte par contumace devant la cour d'assises de Paris contre Aloïs Brunner, le chef du camp de Drancy, vient de retrouver le «fichier des juifs » que l'on croyait perdu ou détruit et qu'il compte en demander la saisie au juge d'instruction en charge du dossier. D’après cet

\footnotetext{
10. Des archives en France, l'activité de la direction des Archives de France et des services publics d'archives, 2006, p. 99.

${ }^{11 .}$ Outre Sonia Combe, KAHN (Annette), Le Fichier, Paris, Robert Laffont, 1993, XX-235 p.
} 
article, le fichier retrouvé dans les services du secrétariat d’État aux Anciens Combattants est bien le grand fichier des juifs de la préfecture de police de Paris, c'est-à-dire le fichier de recensement général de la population juive de la zone occupée, dressé en application des instructions de l'administration militaire allemande du 27 septembre 1940 et confectionné par déclarations individuelles dans les commissariats de police en octobre 1940. Dans les semaines qui suivent, la polémique enfle rapidement pour toute une série de raisons. D’abord, ce fichier revêt sur le plan mémoriel une importance particulière : c'est grâce à lui que les rafles et les déportations de Juifs, et notamment les grandes rafles du Vélodrome d'hiver en 1942, ont pu avoir lieu dans des conditions d'efficacité administrative certaine. Interrogé à plusieurs reprises sur le sort de ce fichier réputé détruit, le ministère des Anciens Combattants avait par ailleurs toujours affirmé ne pas le posséder. Enfin, on apprend assez rapidement que ce fichier, conservé par le bureau des archives de la sous-direction de la réglementation et des statuts, avait conservé une utilité administrative en servant à l'instruction des demandes de pensions ou de titres de juifs déportés. Cet exemple emblématique d'une utilisation administrative prolongée de documents ayant déjà revêtu une valeur historique soulève une large indignation : «en agissant ainsi (c'est-à-dire en se servant de ce fichier), le ministère des Anciens Combattants assumait une classification des citoyens réalisée par un régime non démocratique. On a retourné ce fichier contre ses victimes parce qu'on ne leur faisait pas confiance, préférant se référer au travail de leurs bourreaux. En l'apprenant, j'ai eu de la cendre dans la bouche » déclare l'historien Marc Ferro ${ }^{12}$. Le lieu n'est pas ici de retracer tous les développements de cette affaire qui devaient aboutir le 5 décembre 1997 au dépôt du fichier juif au Mémorial du martyr juif inconnu en présence de Jacques Chirac ${ }^{13}$. Nous voudrions en revanche souligner un certain nombre de points qui relativisent

\footnotetext{
${ }^{12}$. Le Monde, 16 novembre 1991.

13. Pour une vision d'ensemble : JOINET (Louis), « Affaire dite du "Fichier des Juifs" », Le Monde juif, avril-juin 1991, n 142, p. 84-105; POZNANSKI (Renée), « Le fichage des Juifs de France pendant la Seconde Guerre mondiale et l'affaire du fichier juif », La Gazette des archives, 1997, n 177-178, p. 250-270 ; JOLY (Laurent), L'Antisémitisme de bureau. Enquête au cœeur de la préfecture de Police de Paris et du commissariat général aux Questions juives (1940-1944), Paris, Grasset, 2011, p. 316-345. Sur l'origine du fichier: GRAND (Philippe), « Le Fichier juif : un malaise (réponse au rapport Rémond remis au Premier ministre le 3 juillet 1996)", Revue d'histoire de la Shoah, 1999, n 167, p. 53-101; AZÉMA (Jean-Pierre), "Le "fichier juif": réponse à Philippe Grand ", Revue d'histoire de la Shoah, 2000, n 169, p. 249-256.
} 


\section{Invisibilité des archivistes, défaite des historiens ?}

Tout au long de l'affaire les archivistes ont eu la désagréable impression d'être incompris et inaudibles et de ne pas avoir accès aux médias, contraints de confier leur défense à des historiens occupant le devant de la scène. La place et le rôle de ces derniers ne doivent pas pour autant être surestimés. Certes quelques historiens s'expriment régulièrement dans la presse et sur les ondes: René Rémond, Marc Ferro, Jean-Pierre Azéma, François Bédarida, Henry Rousso, Pierre Vidal-Naquet, André Kaspi. Certes, c'est à une commission présidée par l'historien René Rémond, par ailleurs président du Conseil supérieur des archives, que le ministre de la Culture Jack Lang confie le 7 avril 1992 le soin d'étudier la faisabilité juridique du dépôt révocable du fichier au Mémorial du martyr juif inconnu (actuel Mémorial de la Shoah) précédemment préconisé par la Commission nationale de l'informatique et des libertés. Composée de deux autres historiens, JeanPierre Azéma et André Kaspi, d'une archiviste (Chantal de Tourtier-Bonazzi, conservatrice de la section contemporaine) et du président du Conseil représentatif des institutions juives de France (Jean Kahn), la commission recommande dans son rapport définitif remis au ministre le 3 juillet 1996 la conservation du fichier aux Archives nationales, conformément à la réglementation, dans des salles particulières formant un lieu de mémoire. Un microfilm serait remis au Centre de documentation juive contemporaine, tandis que quelques fiches seraient exposées au Mémorial juif. Mais elle n'est pas suivie : dès le 6 juillet 1996, Serge Klarsfeld accuse la commission Rémond dans un article publié par Le Monde d'avoir été formée dans le but de permettre aux Archives nationales de conserver ce fichier et demande au gouvernement de ne pas suivre l'avis de la commission sur le versement du fichier aux Archives nationales, considérant que la Shoah, dont fait partie le recensement de 1940, transcende l'aspect national par l'exceptionnalité d'une tragédie qui englobe les Juifs d'Europe, massacrés non pas en tant que citoyens de leurs pays respectifs, mais pour leur crime d'être nés juifs. C'est cette 
tribune dans Le Monde pour dénoncer le fait que le président de la République «accepte que la patrimoine historique de la nation, et en particulier celui des heures les plus sombres, cesse d'être national ». Et de craindre que les descendants des Vendéens blancs ne réclament le transfert à Cholet des documents ayant trait aux colonnes infernales, ou que le Musée du Désert exige les archives des camisards traqués pour leur foi par les armées du roi.

Plus surprenant, les conclusions de la commission sont contestées y compris sur le terrain de l'analyse historique, là même où sa composition aurait dû suffire à lui assurer une sorte d'infaillibilité admise de tous. Dès le 28 décembre 1992, René Rémond adresse au ministre de la Culture un bref rapport d'étape qui affirme de manière péremptoire que le fichier découvert au secrétariat d'État aux Anciens Combattants n'est pas le fichier général du recensement des Juifs, issu de l'ordonnance des autorités d'occupation du 27 septembre 1940 : «Nous estimons donc être en mesure d'affirmer que le fichier dont la prétendue découverte a soulevé une vive émotion a été détruit, ce qui rend partiellement sans objet une partie de la controverse. » Cette thèse est immédiatement contredite avec force par Serge Klarsfeld. On a pu analyser la polémique comme l'antagonisme de deux postures, la «posture mandarinale», celle du "savoir historique" contre les approximations militantes », face à la «posture militante », celle de la « défense de la “vérité" contre "l'histoire officielle" »" ${ }^{14}$ Il est vrai que Serge Klarsfeld s'est à plusieurs reprises plaint d'avoir dû, plus que tout autre historien, multiplier les stratégies pour contourner les difficultés d'accès aux archives administratives « officielles »" Mais il y a plus. Deux conceptions des archives s'opposent et peinent à se comprendre, celles de ceux qui ont d'abord pour fonction d'élaborer des systèmes explicatifs du passé et celui dont le chef-d'œuvre réside dans la reconstitution patiente des listes de déportés: un

\footnotetext{
14. JOLY (Laurent), L’Antisémitisme de bureau. Enquête au cœur de la préfecture de Police de Paris et du commissariat général aux Questions juives (1940-1944), Paris, Grasset, 2011, p. 336-343.

${ }^{15}$. « Le fichier juif. Table ronde », La Gazette des archives, 1997, $n^{\text {os }}$ 177-178, p. 241-249.
} 

rapport à la psychologie de la communauté juive »: "Quel est l'enfant de déporté qui n'aurait voulu disposer d'une photocopie de la fiche de son père, de sa mère, de ses frères et sœurs? ». En cette occasion, il situe explicitement son action dans un vaste projet qui met les victimes et leurs descendants au cœur : « apaiser des douleurs toujours vivaces en expliquant le destin, en prodiguant des traces écrites de ce destin, en réintégrant la tragédie individuelle dans le drame collectif; en permettant à la mémoire de se perpétuer quand les petits-enfants et leurs descendants peuvent et pourront recevoir les preuves documentaires du passage de leur famille à travers ce cataclysme de I'histoire que fut la Shoah ${ }^{17}$.

Il est d'ailleurs frappant de constater combien le débat a dès le début dépassé la sphère archivistico-historique et combien les tentatives pour l'y faire retourner en le respécialisant ou en le re-technicisant ont été vouées à l'échec. Les auditions menées par la CNIL à la fin de l'année 1991 sur le sort du fichier alors provisoirement déposé aux Archives nationales sont de ce point de vue très significatives, ne serait-ce qu'à travers le statut des personnes entendues. Sur la trentaine de personnalités sollicitées, les historiens ne sont que quatre (François Bédarida, Marc Ferro, Pierre Vidal-Naquet, André Kaspi) tandis que les représentants de la communauté juive, des églises ou des mouvements des droits de l'homme sont majoritaires : directeur du Consistoire central, secrétaire du comité épiscopal pour les relations avec le judaïsme de l’Église catholique, Conseil représentatif des institutions juives de France, Fédération protestante de France, Grande mosquée de Paris, grand rabbin de France, président de la Ligue des droits de l'homme, directeur général du Fonds social juif unifié, Amicale des anciens déportés juifs de France, Grande Loge de France, Grand Orient de France, Ligue internationale contre le racisme et l'antisémitisme (LICRA). Un temps, la loi sur les archives de 1979 connaît une

\footnotetext{
${ }^{16}$. Le Mémorial de la déportation des Juifs de France : listes alphabétiques par convois des Juifs déportés de France, historique des convois de déportation, statistiques de la déportation des Juifs de France, 1978.

${ }^{17}$. KAHN (Annette), Le Fichier, Paris, 1993, p. X.
} 
brutale célébrité et, tandis que, sur les ondes, on peut entendre le ministre de l'Environnement Brice Lalonde estimer qu'il conviendrait de brûler des archives susceptibles de nuire encore à l'avenir ${ }^{18}$, la CNIL fait preuve d'une étonnante inventivité archivistique envisageant successivement toutes les hypothèses quant au sort du fichier, y compris les plus irréalistes : anonymisation du fichier avant versement aux Archives nationales, versement pur et simple aux Archives nationales, maintien au secrétariat d'Etat aux Anciens Combattants, dispersion du fichier dans d'autres fichiers tels celui des déportés, internés et travailleurs, versement au Centre de documentation juive contemporaine (CDJC), versement aux Archives nationales et au CDJC, avec copies réciproques etc.

\section{Indemnisations individuelles et historiographie}

Si l'affaire du fichier juif a confirmé la complexité du rapport collectif à l'histoire de la seconde guerre mondiale et révélé l'intensité du lien qui peut unir individus et documents d'archives, la question de la spoliation des juifs de France et de leur incomplète indemnisation offre, dans une chronologie significativement resserrée, une autre attestation de cette reconfiguration en fournissant l'exemple, nous semble-t-il assez rare, d'explosion d'un champ historiographique à partir d'un besoin à la fois de mémoire et de droit, de nature individuelle en tout cas.

En 1996, une enquête menée par une journaliste sur la partie sud du Marais, « l'îlot 16 », en bordure du vieux quartier juif de Paris, suscite scandale et indignation. Dans son ouvrage Domaine privé, Brigitte Vital-Durand dénonce non seulement les abus auxquels la gestion du domaine privé de la ville de Paris donne lieu comme venait de le rappeler en juin 1995 l'affaire de l'appartement avantageusement mis à la disposition du fils d'Alain Juppé, mais aussi et surtout les conditions historiques de constitution de ce domaine: une partie proviendrait de biens juifs confisqués et non restitués ${ }^{19}$. Le 28 octobre 1996, le conseil municipal de Paris décide de suspendre toute vente de logement tant que leurs

\footnotetext{
${ }^{18}$. « Il faut mettre le feu à tout cela », Le Quotidien, 15 novembre 1991.

${ }^{19}$. VITAL-DURAND (Brigitte), Domaine privé, Paris, First, 1996, 274 p.
} 
date du 25 mars 1997. Le grand public découvre alors l'ampleur de la spoliation économique qui a frappé les Juifs de France pendant la seconde guerre mondiale et notamment les mécanismes d'aryanisation des entreprises. Ces processus n'étaient bien sûr pas ignorés des historiens et des travaux existaient. Ils ne suffirent pas à dispenser la commission de se livrer à un véritable travail d'archives et d'histoire, nécessaire à l'accomplissement de sa mission. Dans la foulée, on note un fort accroissement de la production historique: 110 publications recensées entre 1997 et $2009^{20}$. L'impulsion donnée à la recherche par la demande sociale est ici incontestable. Les archives ont été au cœur de ce mouvement.

\section{Vers l'histoire de soi}

L'idée que la guerre et les grands événements historiques puissent créer des troubles psychologiques sur ceux qui les ont vécus n'est pas nouvelle. À grands traits, on pourrait dire qu'elle s'impose aux lendemains de la première guerre mondiale. L'histoire de cette prise en considération de l'aliénation de guerre est désormais largement défrichée par les historiens de la sortie de guerre ${ }^{21}$. L'idée d'une transmission du trauma et du stress post-traumatique à travers les générations est sans doute plus récente et contestée $^{22}$. Françoise Davoine et Jean-Max Gaudillière ont placé au cœur de leur pratique psychanalytique non seulement les victimes de la folie des guerres mais encore «les descendants qui ont eu la charge de transmettre, de génération en génération, des bouts de temporalité gelés ${ }^{23}$. Ce qui nous paraît plus nettement émergent, du moins en

\footnotetext{
${ }^{20}$. ANDRIEU (Claire), «Ecrire l'histoire des spoliations antisémites (France, 1940-1944)», Histoire@Politique. Politique, culture, société, nº 9, septembre-décembre 2009.

21. TISON (Stéphane), Comment sortir de la guerre? Deuil, mémoire et traumatisme (1870-1940), Rennes, Presses universitaires de Rennes, 2011, $424 \mathrm{p}$.

22. KAËS (René), FAIMBERG (Haydée), ENRIQUEZ (Micheline), sous la dir., Transmission de la vie psychique entre générations, Paris, Dunod, 2003, VIII-208 p.

23. DAVOINE (Françoise), GAUDILLIÈRE (Jean-Max), Histoire et trauma. La folie des guerres, Paris, Stock,
} 
termes de prise de conscience et de théorisation, c'est la place du document d'archives lui-même et l'apparition d'une relation spécifique entre le sujet et «l'archive qui guérit »" face aux «écroulements du monde ${ }^{25}$ dont se sont échappés ceux qui souffrent psychologiquement.

\section{Trauma, récit de vie et passion des archives ${ }^{26}$}

Pendant quatre ans, à partir de 2002, Yoram Mouchenik, psychologue clinicien, a suivi les activités de l'association Mémoires du convoi $n^{\circ} 6$ dédiée à la recherche des enfants, familles, amis des déportés du convoi 6 et à la transmission de leur mémoire ${ }^{27}$. Il participe à leurs réunions, leurs assemblées, leurs voyages, leurs expositions, leurs recherches. Formé d'enfants cachés en France pendant l'Occupation, le groupe s'est constitué dans la volonté d'honorer et de transmettre la mémoire de leurs parents par l'écriture d'un livre. Dans l'analyse à laquelle il se livre du travail de deuil fondé sur ce projet de transmission, Y. Mouchenik note à plusieurs reprises la place de la recherche du document d'archives, support sinon principal du moins central des nombreuses problématiques psychologiques qu’il rencontre et étudie: question du deuil différé, injonction paradoxale de la sépulture malgré l'absence de corps, filiation et réinscription dans une chaîne généalogique, appétit insatiable de savoir sur l'histoire des parents déportés, culpabilité liée à la survivance, interrogations des enfants devenus parents face à la nécessité de transmission d'une histoire familiale, processus auto-thérapeutiques pour gérer une enfance saccagée. Sans pouvoir toujours suivre l'auteur jusqu'au bout du terrain clinique, on ne peut manquer d'être frappé et convaincu par la force des témoignages recueillis. Gérard, membre fondateur de l'association a connu son père,

2006, p. 41.

${ }^{24}$. CYRULNIK (Boris), Autobiographie d'un épouvantail, Paris, Odile Jacob, 2008, p. 12-15.

${ }^{25}$. DAVOINE (Françoise), GAUDILLIÈRE (Jean-Max), Histoire et trauma. La folie des guerres, op. cit., p. 31.

${ }^{26}$. «Entre mémoire et histoire, filiation saccagée, trauma, récit de vie et passion des archives », intervention de Yoram Mouchenik devant la Conférence internationale supérieure d'archivistique, Paris, 16 novembre 2012.

${ }^{27}$. MOUCHENIK (Yoram), « De la disparition au deuil chez les orphelins de la Shoah », L'Autre, 2004, vol. 5, p. 129-141. 
archives les intéressait. C'est devenu une sorte de révélation. » La fréquentation des archives est un tournant dans sa vie et se transforme en impératif : "Dès que j'ai mis le nez là-dedans, je me suis senti dominé par cela. C'est quelque chose qui me domine, c'est ce que je dois faire. » Comme d'autres membres de l'association, il décrit bien le pouvoir d'évocation, de présentification et de matérialisation probatoire du document d'archives : «Les mots, cela suffit, il faut regarder. Cela passe par l'archive. Mon grand-père était marchand forain. Aux Archives nationales, je regarde s'il est aryanisé et je trouve son dossier, avec des courriers où l'administrateur provisoire insiste beaucoup pour être rémunéré. Tu n’es plus dans les mots, mais dans le réel, même si ça s'est passé, il y a longtemps, tu n'es plus dans les fantasmes et l'imagination. [... ] Tout d'un coup, tu as sous les yeux des éléments matériels qui décrivent la trajectoire de ta famille ${ }^{28}$

La lecture de livres d'histoire pourrait-elle avoir, à elle-seule, le même effet de «métabolisation du vécu traumatique ${ }^{29}$ et de « ré-ordonnancement de la succession des générations ${ }^{30}$. Probablement non. Le processus d'historicisation dont il s'agit n'est pas vraiment de l'histoire, ou du moins n'est pas de l'histoire au sens de discours scientifique fondé sur l'analyse des forces sociales et visant à l'établissement d'une forme de compréhension du passé en progrès constant. Il s'agit plutôt de l'élaboration d'un récit de vie qui permet une reconfiguration du moi, « d'une mise en récit par des adultes d'un vécu infantile tragique dont les séquelles traumatiques continuent à se faire entendre ${ }^{31}$. La mobilisation ou l'accumulation des souvenirs biographiques est ici clairement finalisée comme une manière de « l'agir mémoriel » visant à une « réinscription » dans l’histoire de

\footnotetext{
28. MOUCHENIK (Yoram), "Ce n'est qu'un nom sur une liste, mais c'est mon cimetière ». Traumas, deuils et transmission, chez les enfants juifs cachés en France pendant l'Occupation, Paris, La Pensée sauvage, 2006, p. $153-157$.

29. MOUCHENIK (Yoram), « Ce n'est qu'un nom sur une liste, mais c'est mon cimetière »..., op. cit., p. 127.

30. MOUCHENIK (Yoram), «Ce n'est qu'un nom sur une liste, mais c'est mon cimetière »..., op. cit., p. 169.

31. MOUCHENIK (Yoram), « Ce n'est qu'un nom sur une liste, mais c'est mon cimetière »..., op. cit., p. 26.
} 
sa famille et de son groupe social ${ }^{32}$. Bien évidemment, toutes les trajectoires individuelles sont possibles et il n'est pas rare que des véritables profils «d'archivistes-historiens » émergent ${ }^{33}$. Parfois même, hasard ou nécessité, celui qui enquête est historien de métier ; il entend même faire de son enquête un livre d'histoire. Entre charge émotionnelle et nécessité scientifique, on touche à l'ultime de la démarche et alors, mais alors seulement, c'est le travail d'histoire qui se pare d'une dimension thérapeutique. On songe à Ivan Jablonka et à son travail conduit dans le cadre très académique d'une habilitation à diriger des recherches sur ses grands-parents Matès et Idesa, juifs communistes réfugiés en France en 1937, morts en déportation en 1943. On a crié au chef-d'œuvre. A-t-on bien vu combien, au passage, le document d'archives y est installé dans un statut autre que celui, traditionnel, de source historique: «Je l'ai sous les yeux, ce registre d'écrou exhumé aux Archives de la Préfecture de police, intitulé “Voie publique-Étrangers" et je les vois, "Feder Ideta, 11 rue du Pressoir, refus de séjour" et "Jablonska Matès, idem" au bas des pages 390-391 [...], je les vois et je sens leur peur. [...] Je donne rendez-vous un matin à mon père aux Archives de la ville de Paris, près de la porte des Lilas. [... ] Nous passons ensuite au registre de la $16^{\mathrm{e}}$ chambre du tribunal correctionnel de la Seine. Nous tournons les pages fébrilement. Émotion : il est là ». ${ }^{34}$

\section{Nouveaux usages des archives}

Cette approche émotionnellement individualisée du document d'archives reste difficile à cerner quantitativement : dans les salles de lecture des services d'archives rien ne distingue le lecteur historien du lecteur en quête de son histoire et les catégories du formulaire d'inscription dans les services publics d'archives invitant l'usager à s'identifier comme généalogiste, scientifique ou usager effectuant des recherches à caractère administratif ou juridique ne sont pas d'un grand secours. Quelques indices peuvent néanmoins être réunis quant à la dispersion de ce type de comportement.

\footnotetext{
32. LEMÉE-GONÇALVES (Carole), Daniel Galay (Daniel), «L'Après-Shoah: des traumas aux processus de réinscription », Face à face, regards sur la santé, 2003, n 5, p. 83-96.

33. MOUCHENIK (Yoram), «Ce n'est qu'un nom sur une liste, mais c'est mon cimetière »..., op. cit., p. 25.

${ }^{34}$. JABLONKA (Ivan), Histoire des grands-parents que je n'ai pas eus : une enquête, Paris, Seuil, 2012, p. 163.
} 
Sur le Web notamment, listes de diffusion, groupes de discussion, blogs, comptes Twitter, sites familiaux sont un lieu privilégié du partage des documents d'archives pour tous ceux qui cherchent à forger leur compréhension d'un «bout d'histoire échappé à l'Histoire » ${ }^{35}$. "Yahoo group France » héberge tout un éventail de groupes de discussion touchant à divers aspects de la seconde guerre mondiale et qui font une place particulière aux documents d'archives, qu'ils soient écrits ou iconographiques. Le groupe "Stalag » fédère un ensemble d'autres groupes qui se consacrent aux camps de prisonniers pendant la seconde guerre mondiale ${ }^{36}$. Les membres, souvent des descendants de prisonniers, échangent des renseignements sur les services d'archives, des conseils de recherche, des listes de prisonniers, des avis de recherche, des documents, des photographies. Fondé en avril 2007, il rassemble aujourd'hui 1200 membres. À lui seul, le groupe Stalag 1A (Stalags de Prusse orientale), revendique 65000 visites depuis sa fondation en $2007^{37}$. Certains groupes affichent un positionnement militant, en réaction à une écriture de l'histoire dénoncée comme trop officielle. Le groupe «Ardennais réfugiés » entend faciliter les échanges entre tous les évacués en collectant les « récits, anecdotes et photos permettant à chacun de retrouver ses ancêtres et d'identifier des lieux d'exil» afin que cette «partie du patrimoine familial » soit mieux connue ${ }^{38}$. «Victimes de guerre 39-45, les victimes et leurs parcours » est dédié à «toutes les personnes désirant témoigner ou effectuer des recherches sur les travailleurs forcés en pays ennemi, les personnes civiles internées, déportées, dénoncées... ${ }^{39}$. Ouvert en octobre 2007, le groupe reçoit notamment les demandes de renseignements de descendants soucieux de faire « leur devoir de mémoire » : « Je me présente, je suis belge, marié et père de 3 enfants. Je viens de découvrir de vielles photos de captivité de mon grand-père Modeste Dejong né le 24.08.1908 à Hodimont (Verviers) et décédé 25.02.1987. Le sujet de sa captivité étant tabou à la maison, je n'ai que de faibles renseignements,

\footnotetext{
35. DAVOINE (Françoise), GAUDILLIÈRE (Jean-Max), Histoire et trauma. La folie des guerres, op. cit., p. 37.

${ }^{36}$. [En ligne], disponible sur http://fr.groups.yahoo.com/group/stalag/ (consulté le 14 mars 2013).

37. [En ligne], disponible sur http://fr.groups.yahoo.com/group/Stalag_PO (consulté le 14 mars 2013).

${ }^{38}$. [En ligne], disponible sur http://fr.groups.yahoo.com/group/ardennaisrefugies/ (consulté le 14 mars 2013 ).

39. [En ligne], disponible sur http://fr.groups.yahoo.com/group/Victimes-Guerre-39_45/ (consulté le 14 mars 2013).
} 
mais peut-être l'un d'entre vous pourra-t-il étoffer mes données. [...]Mon but est d'essayer de retrouver ce qu'il a pu vivre et en faire un devoir de mémoire pour ses arrière-petits-enfants ${ }^{40}$. Les histoires familiales marquées par la Shoah peuvent déboucher sur la constitution de sites familiaux réservés au noyau familial. « Elie et Pierre Feifer » créé en 2006 ne rassemble que 9 membres. Il se revendique de la tradition juive du livre du souvenir (yisker-buch) et veut « relier les vivants aux morts dans la continuité ininterrompue du temps", ses auteurs ayant conscience de leur responsabilité de «passeurs provisoires de témoignages familiaux transmis à d'autres » ${ }^{41}$.

Les expositions d'archives sont un autre lieu propice à l'exploration du dialogue entre des documents de la seconde guerre mondiale et des publics qui ne sont pas exclusivement mus par la volonté de faire de l'histoire mais plutôt par le désir d'apprendre et de comprendre en famille. Les études par questionnaire ou entretiens auprès des visiteurs demeurent rares dans les expositions d'archives. Les remarques et commentaires consignés dans les livres d'or mis à disposition des visiteurs sont une autre voie d'approche. Une étude menée dans le cadre de l'exposition 1942 en Anjou proposée par les archives départementales de Maine-et-Loire de septembre en janvier 2013 confirme la prégnance d'un rapport individualisé et affectif aux documents d'archives ${ }^{42}$. Alors que les concepteurs de l'exposition entendaient, du moins si l'on en croit leur communication écrite autour du projet, s'en tenir à un discours essentiellement historique $^{43}$, les visiteurs s'en emparent à l'aune de leur histoire personnelle et insistent sur la puissance émotionnelle des documents exposés: "Merci pour cette exposition, particulièrement émouvante pour un petit bonhomme qui avait 6 ans en 42, et "qui,

${ }^{40}$. [En ligne], disponible sur http://fr.groups.yahoo.com/group/Victimes-Guerre-39_45/message/46 (consulté le 14 mars 2013).

${ }^{41}$. [En ligne], disponible sur http://fr.groups.yahoo.com/group/Elie_Et_Pierre_Feifer/ (consulté le 14 mars 2013).

42. BROCHET (Hugues), CORMIER (Maxime), COUPEVENT (Guillaume), GUÉRINES (Côme de), MASSARD (Nicolas), PERRAULT (Lucie), RUCHAUd (Camille), RULLIER (Benjamin), VALENCIA (Océane), "Les publics des expositions des archives départementales de Maine-et-Loire ", Les expositions d'archives et leurs publics. Bilan, mutations, prospectives, Angers, Journée d'étude du 15 février 2013 organisée par les étudiants du master métiers des archives, à paraître, compte rendu, [en ligne], disponible sur http://alma.hypotheses.org/722 (consulté le 15 mars 2013).

43. [En ligne], disponible sur www.cg49.fr/no_cache/haut/espace.../dossiers-de-presse/?cid. (consulté le 14 mars 2013). 
surtout, ne devait rien dire à personne !" (étant le fils d'un instituteur révoqué pour francmaçonnerie). C'est ainsi que je me revois aujourd'hui... »; « J'avais 9 ans en 1942 ! Je me rappelle... les hivers froids... les engelures... Je suis venue ce jour avec mon petit-fils ! »; «L'exposition nous montre concrètement ce que nous avons appris. C'est émouvant! Le nom de mon père doit être dans le registre du Pré-Pigeon ${ }^{44}$ !»; «Merci pour cette expo ! L'année de ma naissance. Ma petite histoire a besoin de la grande vécue ici et partout en France » ; « L'émotion qui m’a gagné ne m’a pas permis d'écrire dans l’instant ! »

\section{Prises de conscience et adaptations}

Ces évolutions des logiques d'usage ${ }^{45}$ sont perçues par les archivistes et tous les professionnels en lien avec les archives Ils sont contraints d'inventer de nouvelles formes d'accueil des publics et d'accompagnement des cheminements personnels, parfois douloureux, induits par la rencontre avec le document d'archives. Dès son premier rapport d'activité en 2001, la CIVS fait état du besoin « d'écoute et d'humanité » ${ }^{46}$ des requérants ce qui la conduit à structurer une «cellule d'accueil et d'assistance » dont les missions sont formalisées en 2002 autour du «soutien psychologique » face à des "parcours personnels tragiques ${ }^{47}$. L'accompagnement ne porte pas uniquement sur l'instruction administrative du dossier des requérants ou le décryptage des séances de la commission, mais aussi sur la communication médiatisée des documents d'archives rassemblés au cours de la procédure. Symétriquement, c'est également les demandes de renseignements et de consultations liées aux spoliations et à la seconde guerre mondiale qui conduisent à l'ouverture en janvier 2004 d'un bureau de recherches administratives familiales au sein du département de la communication des documents du Centre

\footnotetext{
44. Prison d'Angers.
}

45. POISSENOT (Claude), RANJARD (Sophie), Usages des bibliothèques, approche sociologique et méthodologie d'enquête, Villeurbanne, Presses de l'ENSSIB, 2005, p. 248 et suiv.

46. Commission pour l'indemnisation des victimes de spoliations intervenues du fait des législations antisémites en vigueur pendant l'Occupation, Rapport d'activité de la commission présenté à Monsieur le Premier ministre, Paris, 2001, p. 16.

47. Commission pour l'indemnisation des victimes de spoliations intervenues du fait des législations antisémites en vigueur pendant l'Occupation, Rapport d'activité de la Commission présenté à Monsieur le Premier ministre au titre de l'année 2002, Paris, 2002, p. 5. 
historique des Archives nationales (CHAN). Si la description des missions de cette nouvelle cellule administrative insiste principalement sur les nécessités d'orientation des lecteurs, sur le raccourcissement des délais de réponses et sur la nécessité de coordonner les efforts des différents services d'archives concernés ${ }^{48}$, c'est bien en même temps la question de l'accompagnement individualisé d'usagers qui n'ont pas comme seule caractéristique d'être inexpérimentés en recherche dans les archives mais qui sont directement concernés par les documents qu'ils demandent qui fait irruption dans la pratique des archivistes. Le bureau des recherches administratives familiales des Archives nationales s'inspire du «bureau citoyen» des archives départementales de l'Aude, formule également reprise par les archives départementales de l'Hérault qui, depuis leur installation dans les bâtiments de Pierres vives, la Cité des savoirs et du sport pour tous, dispose d'un espace attenant à la salle de lecture « réservé à l'accueil sur rendez-vous de personnes dont les recherches demandent un accompagnement personnalisé ${ }^{49}$. Dans ces deux cas, l’objectif dépasse nettement les archives de la seconde guerre mondiale.

II n'en reste pas moins très significatif de constater que c'est son expérience comme responsable de ce bureau qui a inspiré à l'archiviste Caroline Piketty un livre au ton personnel tout à fait inédit chez les archivistes : "Je cherche les traces de ma mère ", chronique des archives, paru en 2006. On sait le succès qu'a rencontré cet ouvrage, traduit en allemand dès $2007^{50}$, qui a par exemple inspiré un épisode des Éphémères, spectacle proposé par Ariane Mnouchkine et le Théâtre du soleil ${ }^{51}$. Créé à la Cartoucherie en 2006, il a rassemblé plus de 14000 spectateurs au cours d'une tournée nationale et internationale en 2007 et 2008. On y voit une fonctionnaire des archives accueillir, au milieu des cartons et des boîtes, une femme qui cherche à comprendre l'histoire de sa mère, fille de déportés. La scène prend place dans une œuvre tout entière consacrée aux petites histoires, aux origines, aux souvenirs et à leur rapport avec le vécu et le passé collectifs. Il

\footnotetext{
48. PIKETTY (Caroline), Recherches administratives et familiales au Centre historique des Archives nationales, Paris, janvier 2005, [en ligne], disponible sur http://www.archivesnationales.culture.gouv.fr/ chan/chan/pdf/Panneau\%20d\%27Info\%20BRAF-f\%E9v2005-1.pdf (consulté le 15 mars 2013).

49. [en ligne], disponible sur http://pierresvives.herault.fr/ressource/bureau-citoyen (consulté le 15 mars 2013).

${ }^{50}$. Ich suche die Spuren meiner Mutter

51. « Aux archives », épisode 15 du recueil 2 des Éphémères.
} 
y aurait toute une étude à mener sur la réception du livre de Caroline Piketty. Pour les archivistes, il est certain qu'il fait date. C'est la première fois qu'une archiviste prend la parole pour parler de son expérience professionnelle, ce qui n'est déjà pas si fréquent, d'une manière qui n'est ni historique ni archivistique mais entièrement affective. Non que jusqu'alors les archivistes n'aient jamais ressenti gêne ou compassion face aux morceaux de vies qu'ils étaient amenés à connaître, mais ces moments avaient été tus jusqu'alors. C'est le poids de l'expérience, et sa réitération, qui a libéré la parole. Évoquant les fiches anonymes des enfants internés à Drancy, trop petits pour savoir leur nom, l'auteure avoue son envie de se "laver pour tenter de dissiper l'abominable " ${ }^{52}$. Elle touche du doigt la force insoutenable du document d'archives, au point de parfois interrompre la lecture des originaux pour proposer l'envoi postal de photocopies : « Je n’ai pas souhaité les voir prolonger leur lecture dans mon bureau, sachant combien elle était lourde de sens, et je leur ai proposé de leur faire parvenir la photocopie intégrale de ces documents. ${ }^{53}$ Elle sent bien que sa conception du métier se transforme et que son rôle s'éloigne des rivages assurées de la technique archivistique et de l'histoire: «Du thérapeute, je m'approche sans être vraiment certaine de savoir panser les plaies trop profondes. Je franchis des passerelles, chargée des questions qui me sont posées, soulageant quelque peu ceux qui me les posent en les invitant à passer le gué. ${ }^{54}$ Le choc de professionnalité est telle qu'il autorise, en pensée du moins, toutes les transgressions : « J'ai une tendresse particulière et coupable pour les familles des spoliés. Si d'aventure l'un d'entre eux était sur le point d'emporter une photo, une lettre manuscrite ou une pièce d'identité de son père ou de sa mère, j'avoue que je le comprendrais. ${ }^{55}$

\footnotetext{
52. Caroline Piketty, "Je cherche les traces de ma mère ». Chronique des archives, Paris, Autrement, 2006, p. 106.

53. Caroline Piketty, "Je cherche les traces de ma mère ". Chronique des archives, Paris, Autrement, 2006, p. 81.

54. Caroline Piketty, "Je cherche les traces de ma mère ». Chronique des archives, Paris, Autrement, 2006, p. 102.

55. Caroline Piketty, "Je cherche les traces de ma mère". Chronique des archives, Paris, Autrement, 2006, p. 100.
} 
«Le mort saisit le vif »: cet adage médiéval servait à exprimer l'instantanéité de la dévolution successorale et signifier que la saisine des biens du défunt est acquise le jour même de la mort à son héritier. Pierre Bourdieu l'avait détourné à son profit pour exposer ses conceptions des rapports de l'histoire et de la sociologie ${ }^{56}$. "L'archive saisit le vif » serions-nous tentés de dire en prenant de tout aussi grandes libertés avec le sens initial de la maxime juridique. Nulle immédiateté obligée dans le processus qui met en relation les descendants d'un déporté, d'un prisonnier, d'un réfugié, d'un résistant ou d'un collaborateur, avec les archives qui gardent des traces de leur parcours. La rencontre se fait selon une temporalité aléatoire, dans laquelle cependant le décès d'un parent ou d'un grand-parent n'est pas sans jouer un rôle. C'est bien d'un « saisissement » qu'il s'agit en revanche, à tous les sens du terme: l'appropriation de l'événement historique par le roman familial passe nécessairement par la rencontre avec le document d'archives qui révèle alors toute sa puissance agissante sans échappatoire possible. Observable à propos des archives de la seconde guerre mondiale et des logiques d'usage qu'elles suscitent à grande échelle dans les années 1990, cette orientation individualisée du rapport aux archives n'est pas pour autant spécifique à cette période historique. Bien au contraire, la demande adressée aux archives de la seconde guerre mondiale préfigure, ou simplement traduit un vaste mouvement de relativisation des sollicitations purement historiques du matériau archivistique au profit des stratégies de recherche de soi et de construction de l'individualité ${ }^{57}$.

\footnotetext{
${ }^{56}$. BOURDIEU (Pierre), «Le mort saisit le vif. Les relations entre l'histoire réifiée et l'histoire incorporée », Actes de la recherche en sciences sociales, 1980, vol. 32, $\mathrm{n}^{\text {os }} 32-33$, p. 3-14.

57. MARCILLOUX (Patrice), Les Ego-archives. Traces documentaires et recherches de soi, Rennes, Presses universitaires de Rennes, 2013.
} 
Recebido em: 02/12/2012 Aprovado em: 01/04/2013

Universidade do Estado de Santa Catarina - UDESC

Programa de Pós-Graduação em História - PPGH

Revista Tempo e Argumento

Volume 05 - Número 09 - Ano 2013 tempoeargumento@gmail.com 